\title{
MEAN VALUES AND BANACH LIMITS ${ }^{1}$
}

\section{RALPH A. RAIMI}

I. Introduction. For certain bounded real valued functions on the real line, $\lim _{\alpha}(1 / 2 \alpha) \int_{-\alpha}^{\alpha} f(x+t) d t$ exists uniformly in $x$, and is a constant, $m(f)$. On the space of such functions, the limit $m(f)$ is a translation-invariant positive functional of norm one, and hence generalizes the notion $\lim _{|x| \rightarrow \infty} f(x)$. For bounded sequences, such a functional is often called a Banach limit $\left([1, \mathrm{pp} .83-84]\right.$, and [5]). ${ }^{2}$ Norm preserving extensions of $m(f)$ to wider spaces will still be called Banach limits. In sections II and III the properties of these extensions will be explored, in particular their extreme values, and in section IV a space will be exhibited for which all Banach limits are obtained in this way.

The author wishes to express his appreciation of the assistance of Professor S. Kakutani, to whom Theorem 3 is due, as well as numerous suggestions and conjectures.

II. Preliminaries. Let $E$ be a complete normed linear space of realvalued essentially bounded measurable functions on $R$, the real line, with $\|f\|=$ ess. sup. $\{|f(x)| \mid x \in R\}$, denoted hereafter merely by $\sup _{x}|f(x)|$. That $E$ is actually made up of equivalence classes of such functions will be ignored in the sequel wherever no confusion thus arises. We shall assume further that $E$ contains all bounded uniformly continuous functions, and all translates of functions in $E$, i.e. if $f(x) \in E$, then $f(x+s) \in E$, for any $s \in R$.

Let $E^{\prime}$ be the conjugate space of $E$, and $L(E, E)$ the ring of continuous linear operators on $E$ to $E$. For each $s \in R$ we define an element $T_{s} \in L(E, E)$ by $T_{s}: f(x) \rightarrow f(x+s)$. Denoting by $R^{+}$the set of all positive real numbers, we define for each $\alpha \in R^{+}$the operation $T_{\alpha}: f(x) \rightarrow(1 / 2 \alpha) \int_{-\alpha}^{\alpha} f(x+t) d t$.

Lemma 0 . For any $f \in E, \alpha \in R^{+}, T_{\alpha} f$ is a uniformly continuous function on the real line. In particular $T_{\alpha} \in L(E, E)$ for all $\alpha \in R^{+}$.

Proof. Let $\epsilon>0$ be given, and choose $s \in R$ such that $|s|<\alpha$, and $|s|<(\alpha \epsilon /\|f\|)$. Then

Presented to the Society April 21, 1956; received by the editors January 19, 1956.

1 This paper was written while the author was an Alfred H. Lloyd Postdoctoral Fellow of the Horace H. Rackham School of Graduate Studies of the University of Michigan.

${ }^{2}$ Numbers in brackets refer to the bibliography at the end of the paper. 


$$
\begin{aligned}
\left|T_{\alpha} f(x)-T_{\alpha} f(x+s)\right| & =(1 / 2) \alpha\left|\int_{-\alpha}^{\alpha} f(x+t) d t-\int_{-\alpha}^{\alpha} f(x+t+s) d t\right| \\
& =(1 / 2 \alpha)\left|\int_{-\alpha}^{\alpha} f(x+t) d t-\int_{-\alpha+s}^{\alpha+s} f(x+t) d t\right| \\
& =(1 / 2 \alpha)\left|\int_{-\alpha}^{-\alpha+s} f(x+t) d t-\int_{\alpha}^{\alpha+s} f(x+t) d t\right| \\
& \leqq(2\|f\||s| / 2 \alpha)<\epsilon,
\end{aligned}
$$

independently of $x$.

If we set $\mathfrak{T}=\left\{T_{\alpha} \mid \alpha \in R^{+}\right\}$, and denote the set of translation operators $\left\{T_{s} \mid s \in R\right\}$ by $\mathfrak{A}$, we observe that the elements of $\mathfrak{T} \cup \mathfrak{A}$ commute with each other.

Let $E^{\prime}$ denote the conjugate space of $E$. If $T \in L(E, E), T^{\prime}$ will denote the adjoint operator. $T^{\prime}$ is continuous on $E^{\prime}$ to $E^{\prime}$ when $E^{\prime}$ is given its weak-* topology. This is the only topology on $E^{\prime}$ which will be considered. If $\phi^{\prime} \in E^{\prime}, f \in E,\left(\phi^{\prime}, f\right)$ will denote the value of the functional $\phi^{\prime}$ at the point $f$.

If $x \in R$, and $f$ is a continuous function, we denote by $x^{\prime}$ the "pointfunctional" $x^{\prime}: f \rightarrow f(x)$. If $f \in E$ is an equivalence class of functions containing a continuous representative, we shall define $\left(x^{\prime}, f\right)=f(x)$ for that representative. Now $x^{\prime}$ becomes a functional defined unambiguously on a closed subspace of $E$, and may be extended, with preservation of the norm $\left\|x^{\prime}\right\|=1$, to any of a number of elements of $E^{\prime}$. In what follows, the symbol $x^{\prime}$ will represent any such element of $E^{\prime}$, arising from the number $x \in R$, and the set of all such functionals, as $x$ runs through $R$, will be denoted $R^{\prime}$.

Since, for any $f \in E, T_{\alpha} f$ is continuous (Lemma 0$),\left(x^{\prime}, T_{\alpha} f\right)$ is a well-defined real number. Since $\left(x^{\prime}, T_{\alpha} f\right)=\left(T_{\alpha}^{\prime} x^{\prime}, f\right)$, it follows that $T_{\alpha}^{\prime} x^{\prime} \in E^{\prime}$ is a well-defined functional, no matter which extension of the point-functional corresponding to $x$ is meant by the symbol $x^{\prime}$.

$R^{+}$is a net (or directed set; see [4, pp. $\left.65 \mathrm{ff}\right]$ ) under the usual ordering. Any unbounded subset of $R^{+}$is cofinal under the same ordering. $B$ is a subnet of $R^{+}$if $B C R^{+}$and $B$ is a directed set in its own right, under some other ordering, perhaps, but with the property that given $\alpha \in R^{+}, \beta \in B$, there exists $\gamma \in B$ with $\gamma>\beta$ in the ordering of $B$ and $\gamma>\alpha$ in the ordering of $R^{+}$. In particular, a subnet of $R^{+}$must be unbounded. We shall also call a net any function from a net into a topological space, and use the notation (e.g.) $g=l p_{\beta} f_{\beta}(\beta \in B)$ to mean $g$ is a topological point of accumulation of every terminal section $\left\{f_{\beta} \mid \beta>\beta_{0}\right\}$ of the net $\left\{f_{\beta}\right\}$. If $g=l p_{\beta} f_{\beta}$, then $g=\lim _{\gamma} f_{\gamma}$, where $\left\{f_{\gamma}\right\}$ is some subnet of $\left\{f_{\beta}\right\}$. By way of converse, if $g=l p_{\gamma} f_{\gamma}$, and if $\left\{f_{\gamma}\right\}$ is a subnet of $\left\{f_{\beta}\right\}$, then $g=l p_{\beta} f_{\beta}$. 
We shall denote by $V$ that (closed) subspace of $E$ consisting of all $f \in E$ such that $\lim _{\alpha} T_{\alpha} f$ exists in the norm topology of $E$, and shall use the symbol $f_{1}$ to denote the constant function of $E: f_{1}(x)=1$ for all $x \in R$.

Lemma 1. If $f \in V$, then there exists a number $m(f) \in R$ such that $\lim _{\alpha} T_{\alpha} f=m(f) f_{1}$.

Proof. Let $\lim _{\alpha} T_{\alpha} f(x)=g(x)$. For $\epsilon>0, x \neq 0,|g(x)-g(0)|$ $=\left|\lim _{\alpha}(1 / 2 \alpha) \int_{-\alpha}^{\alpha}(f(x+t)-f(t)) d t\right| \leqq(1 / 2 \alpha)\left|\int_{-\alpha+x}^{\alpha+x} f(t) d t-\int_{-\alpha}^{\alpha} f(t) d t\right|$ $+\epsilon$ for all $\alpha>\alpha_{0}$ suitably chosen. But if $\alpha>|x|$,

$$
\begin{aligned}
\int_{-\alpha+x}^{\alpha+x} f(l) d \iota-\int_{-\alpha}^{\alpha} f(t) d \iota \mid & \\
= & \left|\int_{-\alpha}^{-\alpha+x} f(t) d \iota-\int_{\alpha}^{\alpha+x} f(t) d \iota\right| \leqq 2|x| \cdot\|f\| .
\end{aligned}
$$

Hence $\alpha>\alpha_{0}, \quad|x| \Rightarrow|g(x)-g(0)|<\epsilon+|x|\|f\| / \alpha$ which can be made arbitrarily small. Thus $g$, a constant function, is a multiple of $f_{1}$.

We shall call $m(f)$ the mean value of $f$, for $f \in V$. This is a linear functional on the subspace $V$ of $E$.

Definition 1. $\phi^{\prime} \in E^{\prime}$ is called a mean-value functional if it is a norm-preserving extension of the functional $m(f)$ defined on $V$. Precisely:

(a) $\left\|\phi^{\prime}\right\|=1$,

(b) $f \in V \Rightarrow\left(\phi^{\prime}, f\right)=m(f)$.

We denote by $M^{\prime}$ the set of mean-value functionals.

LEMMA 2.

(a) $\left\|T_{\alpha}\right\|=\left\|T_{s}\right\|=1$ for all $s \in R, \alpha \in R^{+}$,

(b) $\lim _{\alpha}\left\|T_{\alpha} T_{s} f-T_{\alpha} f\right\|=0$, for all $s \in R$,

(c) $\lim _{\alpha}\left\|T_{\alpha} T_{\beta} f-T_{\alpha} f\right\|=0$, for all $\beta \in R^{+}$.

Proof. (a) is obvious. (b) is proved exactly as in the proof of Lemma 2, i.e., for $\alpha>|s|$,

$$
\begin{aligned}
\left(T_{\alpha} T_{s} f-T_{\alpha} f\right)(x) & =(1 / 2 \alpha)\left(\int_{-\alpha+s}^{\alpha+s}-\int_{-\alpha}^{\alpha}\right)(f(t+x) d l) \\
& =(1 / 2 \alpha)\left(\int_{-\alpha}^{-\alpha+s}-\int_{\alpha}^{\alpha+s}\right)(f(t+x) d t)
\end{aligned}
$$

which in absolute value is $\leqq(|s| / \alpha)\|f\|$ for all $x$, and this can be made arbitrarily small as $\alpha \rightarrow \infty$. As for (c): For each $\alpha \in R^{+}$, define $g_{\alpha}(x)=(1 / 2 \alpha)$ for $-\alpha \leqq x \leqq \alpha$, and $g_{\alpha}(x)=0$ otherwise. If convolution 
is defined by $(F * G)(x)=\int_{-\infty}^{\infty} F(t) G(x+t) d t$, then it is clear that $\left(T_{\alpha} T_{\beta}-T_{\alpha}\right) f(x)=\left(g_{\alpha} * g_{\beta} * f-g_{\alpha} * f\right)(x)$. For each $\alpha, g_{\alpha} \in L^{1}$, indeed $\left\|g_{\alpha}\right\|_{1}=1$. Denoting the norm in $E$ for the moment by $\|f\|_{\infty}$, we have the well-known inequality $\left\|\left(g_{\alpha} * g_{\beta}-g_{\alpha}\right) * f\right\|_{\infty} \leqq\left\|g_{\alpha} * g_{\beta}-g_{\alpha}\right\|_{1} \cdot\|f\|_{\infty}$. But a calculation will show that $\lim _{\alpha}\left\|g_{\alpha} * g_{\beta}-g_{\alpha}\right\|_{1}=0$.

\section{The Set $M^{\prime}$.}

Lemma 3. $M^{\prime}$ is convex and compact in the weak-* topology of $E^{\prime}$. If $\phi^{\prime} \in M^{\prime}$ and $f \in E$, and $f(x) \geqq 0$ a.e., then $\left(\phi^{\prime}, f\right) \geqq 0$.

Proof. Convexity and closure of $M^{\prime}$ follow immediately from Definition 1 , and hence compactness because $M^{\prime}$ is a bounded set of $E^{\prime} .\left(\phi^{\prime}, f_{1}\right)=m\left(f_{1}\right)=1$. Now let $0 \leqq f(x) \leqq 1$ a.e. Then $f_{1}-f$ has the same essential bounds. $\left(\phi^{\prime}, f\right)=1-\left(\phi^{\prime}, f_{1}-f\right)$. But $\left\|\phi^{\prime}\right\|=1,\left\|f_{1}-f\right\| \leqq 1$ implies $\left(\phi^{\prime}, f_{1}-f\right) \leqq 1$, i.e. $\left(\phi^{\prime}, f\right) \geqq 0$. For arbitrary positive $f \in E$, the result follows by the homogeneity of $\phi^{\prime}$.

Lemma 4. Let $B \subset R^{+}$be a subnet, and $\left\{y_{\beta}^{\prime} \mid \beta \in B\right\} \subset E^{\prime}$ have the properties $\left\|y_{\beta}^{\prime}\right\|=1$ and $\left(y_{\beta}^{\prime}, f_{1}\right)=1$ for all $\beta \in B$. If $\phi^{\prime}=l p_{\beta}\left\{T_{\beta}^{\prime} y_{\beta}^{\prime}\right\}$, then $\phi^{\prime} \in M^{\prime}$.

Proof. $\quad\left(\phi^{\prime}, f_{1}\right)=l_{p_{\beta}}\left(T_{\beta}^{\prime} y_{\beta}^{\prime}, f_{1}\right)=l_{p_{\beta}}\left(y_{\beta}^{\prime}, T_{\beta} f_{1}\right)=l_{p_{\beta}}\left(y_{\beta}^{\prime}, f_{1}\right)=1$. Since for all $\beta \in B,\left\|T_{\beta}^{\prime} y_{\beta}^{\prime}\right\| \leqq 1$, we have $\left\|\phi^{\prime}\right\| \leqq 1$, hence $\left\|\phi^{\prime}\right\|=1$, and (a) of Definition 1 holds. Now let $f \in V$. Then $\left(\phi^{\prime}, f\right)=l_{p_{\beta}}\left(T_{\beta}^{\prime} y_{\beta}^{\prime}, f\right)$ $=l p_{\beta}\left(y_{\beta}^{\prime}, T_{\beta} f\right)$. For any $\epsilon>0, \exists \beta_{0}$ such that $\left\|T_{\beta} f-m(f) f_{1}\right\|<\epsilon$ for all $\beta>\beta_{0}$. Thus $\left|\left(y_{\beta}^{\prime}, T_{\beta} f-m(f) f_{1}\right)\right|=\left|\left(y_{\beta}^{\prime}, T_{\beta} f\right)-m(f)\right|<\epsilon$. For a suitable $\beta>\beta_{0},\left|\left(\phi^{\prime}, f\right)-\left(y_{\beta}^{\prime}, T_{\beta} f\right)\right|<\epsilon$, and combining the inequalities, $\left|\left(\phi^{\prime}, f\right)-m(f)\right|<2 \epsilon$, and (b) of Definition 1 holds.

Lemma 5. Let $f \in E$, and let $B \subset R^{+}$be a subnet. Let $\left\{u_{\beta}^{\prime} \mid \beta \in B\right\} \subset E^{\prime}$, and $\left\|u_{\beta}^{\prime}\right\| \leqq l$ for all $\beta \in B$. If $\lambda=l_{p_{\beta}}\left(u_{\beta}^{\prime}, f\right)$, there exists some $\phi^{\prime} \in E^{\prime}$ such that $\phi^{\prime}=l_{p_{\beta}} u_{\beta}^{\prime}$ (in the weak-* topology) and $\left(\phi^{\prime}, f\right)=\lambda$.

Proof. Since $\lambda=l p_{\beta}\left(u_{\beta}^{\prime}, f\right)$ there is a subnet of $B$, call it $C$, such that $\lambda=\lim \left\{\left(u_{\gamma}^{\prime}, f\right) \mid \gamma \in C\right\}$. Since $\left\|u_{\gamma}^{\prime}\right\| \leqq 1$, and the unit ball of $E^{\prime}$ is compact in the weak-* topology, there exists $\phi^{\prime}=l_{p_{\gamma}}\left\{u_{\gamma}^{\prime}\right\}$. But then $\left(\phi^{\prime}, f\right)=l p_{\gamma}\left(u_{\gamma}^{\prime}, f\right)=\lim _{\gamma}\left(u_{\gamma}^{\prime}, f\right)=\lambda$.

DEFinition 2. $L^{\prime} \subset E^{\prime}$ will denote the set of all functionals of the form $\phi^{\prime}=l p_{\alpha}\left\{T_{\alpha}^{\prime} x_{\alpha}^{\prime} \mid \alpha \in R^{+}\right\}$, where for every $\alpha, x_{\alpha}^{\prime} \in R^{\prime}$, i.e. $x_{\alpha}^{\prime}$ is a point-functional.

We observe that if $B \subset R^{+}$is a subnet, and $\phi^{\prime}=l p\left\{T_{\beta}^{\prime} x_{\beta}^{\prime} \mid \beta \in B\right\}$, then $\phi^{\prime} \in L^{\prime}$.

Lemma 6. $L^{\prime} \subset M^{\prime}$. 
Proof. Follows directly from Lemma 4, since point-functionals $x_{\beta}^{\prime}$ satisfy the requirements $\left\|x_{\beta}^{\prime}\right\|=1$ and $\left(x_{\beta}^{\prime}, f_{1}\right)=1$.

Lemma 7. Let $f \in E$, and let $\left\{x_{\beta}^{\prime} \mid \beta \in B\right\} \subset R^{\prime}$ be a directed set of point functionals, $B$ a subset of $R^{+}$. If $\lambda=l p_{\beta}\left(T_{\beta}^{\prime} x_{\beta}^{\prime}, f\right)$, then there exists some $\phi^{\prime} \in L^{\prime}$ such that $\left(\phi^{\prime}, f\right)=\lambda$.

Proof. If we set $u_{\beta}^{\prime}=T_{\beta}^{\prime} x_{\beta}^{\prime}$, we find the hypotheses of Lemma 5 are satisfied, and hence $\left(\phi^{\prime}, f\right)=\lambda$. That $\phi^{\prime} \in L^{\prime}$ is evident from the construction.

Theorem 1. Let $f \in E$, and let

$$
\begin{aligned}
& \tau(f)=\liminf _{\alpha} \inf _{x} T_{\alpha} f(x), \\
& \omega(f)=\limsup _{\alpha} \sup _{x} T_{\alpha} f(x) .
\end{aligned}
$$

Then there exists $\phi^{\prime} \in M^{\prime}$ with $\left(\phi^{\prime}, f\right)=\lambda$ if and only if $\tau(f) \leqq \lambda \leqq \omega(f)$. Further, for any such value $\lambda$ a corresponding $\phi^{\prime} \in L^{\prime}$ may be found such that $\left(\phi^{\prime}, f\right)=\lambda$.

Proof. If $\lambda=\tau(f)$, we can choose for each $\alpha>0$ some point $x_{\alpha} \in R$ such that $\left|\inf _{x} T_{\alpha} f(x)-T_{\alpha} f\left(x_{\alpha}\right)\right|<1 / \alpha$. Then clearly $\lambda$ $=\lim \inf _{\alpha} \inf _{x} T_{\alpha} f(x)=\lim \inf _{\alpha} T_{\alpha} f\left(x_{\alpha}\right)$. But $T_{\alpha} f\left(x_{\alpha}\right)=\left(x_{\alpha}^{\prime}, T_{\alpha} f\right)$ $=\left(T_{\alpha}^{\prime} x_{\alpha}^{\prime}, f\right)$, where $x_{\alpha}^{\prime} \in R^{\prime}$, and we have $\lambda=l p_{\alpha}\left(T_{\alpha}^{\prime} x_{\alpha}^{\prime}, f\right)$. By Lemma 7 , there exists $\phi^{\prime} \in L^{\prime}$ such that $\left(\phi^{\prime}, f\right)=\lambda$. Similarly, another $\phi^{\prime} \in L^{\prime}$ can be found such that $\left(\phi^{\prime}, f\right)=\omega(f)$. Now let $\tau(f)<\lambda<\omega(f)$. Then

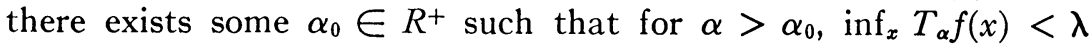
$<\sup _{x} T_{\alpha} f(x)$. Since $T_{\alpha} f(x)$ is a continuous function of $x$, there exists for each $\alpha>\alpha_{0}$ a point $x_{\alpha}$ such that $\lambda=T_{\alpha} f\left(x_{\alpha}\right)=\left(x_{\alpha}^{\prime}, T_{\alpha} f\right)=\left(T_{\alpha}^{\prime} x_{\alpha}^{\prime}, f\right)$. Thus $\lambda=\lim _{\alpha}\left(T_{\alpha}^{\prime} x_{\alpha}^{\prime}, f\right)$, and Lemma 7 applies again. It remains to show that if $\lambda<\tau(f)$, there is no $\phi^{\prime} \in M^{\prime}$ with $\left(\phi^{\prime}, f\right)=\lambda$, for a similar argument will show $\lambda>\omega(f)$ is also not a possible value. Suppose then $\lambda<\tau(f)$, but that $\left(\phi^{\prime}, f\right)=\lambda, \phi^{\prime} \in M^{\prime}$. Then there exists some $\alpha_{0} \in R^{+}$and some $\delta>0$ such that $\lambda \leqq T_{\alpha} f(x)-\delta$ for all $x \in R$, all $\alpha>\alpha_{0}$. Then $T_{\alpha} f(x)-\delta f_{1}(x)-\lambda f_{1}(x) \geqq 0\left(x \in R, \alpha>\alpha_{0}\right)$. By Lemma 3 $\left(\phi^{\prime}, T_{\alpha} f-f_{1}-\lambda f_{1}\right) \geqq 0$. But $\left(\phi^{\prime}, T_{\alpha} f\right)=\left(\phi^{\prime}, f\right)$ for any $\phi^{\prime} \in M^{\prime}$, and we have $\left(\phi^{\prime}, f\right)-\delta\left(\phi^{\prime}, f_{1}\right)-\lambda\left(\phi^{\prime}, f_{1}\right)=-\delta \geqq 0$ which is a contradiction.

Theorem 2. $M^{\prime}$ is the closed convex hull of $L^{\prime}$.

Proof. Since $M^{\prime}$ is convex and compact, it is sufficient (by the Krein-Milman theorem) to show $L^{\prime}$ contains all the extreme point of $M^{\prime}$. Equivalently [3, Theorem 1] it suffices to show, for every $f \in E$, that $\sup \left\{\left(\phi^{\prime}, f\right) \mid \phi^{\prime} \in M^{\prime}\right\}=\sup \left\{\left(\phi^{\prime}, f\right) \mid \phi^{\prime} \in L^{\prime}\right\}$. But Theorem 1 showed both these numbers to be $\omega(f)$. 
The expressions for $\tau(f)$ and $\omega(f)$ in Theorem 1 can be sharpened in appearance, as well as expressed in other forms, via the following theorem. We shall denote by $V_{0}$ that subspace of $V$ composed of all $f \in V$ such that $m(f)=0$, and observe that, by Lemma 2(c), for every $f \in E, \alpha \in R^{+}, T_{\alpha} f-f \in V_{0}$. Also, any function in $E$ vanishing outside a compact subset of $R$ is in $V_{0}$.

THEOREM 3. Let

$$
\begin{aligned}
& A=\limsup _{\alpha} \sup _{x} T_{\alpha} f(x), \\
& B=\lim _{\alpha} \inf \limsup _{|x| \rightarrow \infty} T_{\alpha} f(x), \\
& C=\inf _{g \in V_{0}} \sup _{x}(f(x)-g(x)), \\
& D=\inf _{g \in V_{0}} \limsup _{|x| \rightarrow \infty}(f(x)-g(x)), \\
& G=\inf _{g \in V}(m(g)+\|f-g\|)
\end{aligned}
$$

for any $f \in E$. Then $A=B=C=D=G=\omega(f)$. In particular, lim $\sup _{\alpha}$ and $\lim \inf _{\alpha}$ in the expressions for $A$ and $B$ may both be replaced by $\lim _{\alpha}$.

Proof. Clearly $A \geqq B$ and $C \geqq D$. To show $A \geqq C$, set $g_{\alpha}=f-T_{\alpha} f$ for each $\alpha \in R^{+}$. Then $g_{\alpha} \in V_{0}$, and $\sup _{x} T_{\alpha} f(x)=\sup _{x}\left(f(x)-g_{\alpha}(x)\right) \geqq C$ for all $\alpha$. To show $B \geqq D$, the procedure is the same. Then $A=B$ $=C=D$ follows if we show $A \leqq D$. Let $\epsilon>0$, and let $g \in V_{0}$ be chosen such that

$$
\limsup _{|x| \rightarrow \infty}(f(x)-g(x))<D+\epsilon .
$$

Then it is possible to find some $h(x) \in E$ which vanishes outside a finite interval (hence $h \in V_{0}$ ) such that $\sup _{x}(f(x)-g(x)-h(x)$ ) $<D+\epsilon$. Then for all $\alpha \in R^{+}, \sup _{x}\left[T_{\alpha} f(x)-T_{\alpha} g(x)-T_{\alpha} h(x)\right]<D+\epsilon$. But $\lim _{\alpha}\left(T_{\alpha} g(x)+T_{\alpha} h(x)\right)=0$ uniformly in $x$, hence there exists $\alpha_{0} \in R^{+}$such that for all $\alpha>\alpha_{0}$, $\sup _{x} T_{\alpha} f(x)<D+2 \epsilon$, and hence $A \leqq D+2 \epsilon$. Finally we show $A=G$. First we assume $f(x) \geqq 0$. Now $A=\omega(f)=\left(\phi^{\prime}, f\right)$ for some $\phi^{\prime} \in M^{\prime}$. For any $g \in V,\left(\phi^{\prime}, f-g\right) \leqq\|f-g\|$, or $A=\left(\phi^{\prime}, f\right) \leqq\left(\phi^{\prime}, g\right)+\|f-g\|$, i.e. $A \leqq m(g)+\|f-g\|$ for all $g \in V$, or $A \leqq G$. Now if $A<G$, there exists $\alpha_{0}$ such that for all $g \in V$, $\sup _{x} T_{\alpha} f(x)<m(g)+\|f-g\|$. But if $g=f-T_{\alpha_{0}} f, m(g)=0$, and we obtain $\sup _{x} T_{\alpha_{0}} f(x)<\left\|T_{\alpha_{0}} f\right\|$, whereas since $f(x) \geqq 0$, $\sup _{x} T_{\alpha_{0}} f(x)$ $=\left\|T_{\alpha_{0}} f\right\|$. Thus $A=G$ if $f(x) \geqq 0$. For an arbitrary $f \in E,\left(f+\|f\| f_{1}\right)(x)$ $\geqq 0$. An easy calculation shows that 
$\limsup \sup _{\alpha} T_{\alpha}\left(f(x)+\|f\| f_{1}(x)\right)=\|f\|+\limsup _{\alpha} \sup _{x} T_{\alpha} f(x)$,
$\inf _{o \in V}\left(m(g)+\|f+\| f\left\|f_{1}-g\right\|\right)=\|f\|+\inf _{o \in V}(m(g)+\|f-g\|)$

from which the conclusion follows. In the second calculation it is necessary to observe that $g-\|f\| f_{1}$ runs through all of $V$ as $g$ does.

In exactly similar style, the following theorem gives equivalent expressions for $\tau(f)$. The proof will be omitted.

THEOREM 4. Let

$$
\begin{aligned}
& A=\liminf _{\alpha} \inf _{x} T_{\alpha} f(x), \\
& B=\limsup _{\alpha} \liminf _{|x| \rightarrow \infty} T_{\alpha} f(x), \\
& C=\sup _{g \in V_{0}} \inf _{x}(f(x)-g(x)), \\
& D=\sup _{g \in V_{\bullet}} \liminf _{|x| \rightarrow \infty}(f(x)-g(x)), \\
& G=\sup _{g \in V}(m(g)-\|f-g\|)
\end{aligned}
$$

for any $f \in E$. Then $A=B=C=D=G=\tau(f)$. In particular lim inf ${ }_{\alpha}$ and $\lim \sup _{\alpha}$ in the expression for $A$ and $B$ may be replaced by $\lim _{\alpha}$.

\section{Uniformly continuous functions}

Definition 3. An element $\phi^{\prime} \in E^{\prime}$ will be called a Banach Limit when

(a) $\left\|\phi^{\prime}\right\|=1$,

(b) $\left(\phi^{\prime}, f_{1}\right)=1$,

(c) $\left(\phi^{\prime}, T_{s} f\right)=\left(\phi^{\prime}, f\right)$, for all $f \in E, s \in R$.

The set of Banach Limits will be denoted $B^{\prime}$.

Theorem 5. $M^{\prime} \subset B^{\prime}$; i.e. every mean value functional is a Banach Limit.

Proof. (a) and (b) are immediate from (a) and (b) of Definition 1. To show $\left(\phi^{\prime}, T_{s} f-f\right)=0$, we observe that $\lim _{\alpha}\left\|T_{\alpha}\left(T_{s} f-f\right)\right\|=0$ (Lemma $2(\mathrm{~b})$ ), i.e. $T_{s} f-f \in V$ and $m\left(T_{s} f-f\right)=0$, and apply Definition $1(\mathrm{~b})$.

THEOREM 6. If $E$ is the space of bounded uniformly continuous real functions on $R$, then $M^{\prime}=B^{\prime}$.

Proof. If $f$ is uniformly continuous $T_{\alpha} f(x)$ is uniformly approximated by its Riemann sums, which is to say $\left\|T_{\alpha} f-\sum a_{i} T_{s_{i}} f\right\|<\epsilon$ for 
arbitrary $\epsilon$ and suitable choice of the convex combination $\sum a_{i} T_{s_{i}} f$. Now let $\phi^{\prime} \in B^{\prime}$, and let $f \in V$; we must show $\left(\phi^{\prime}, f\right)=m(f)$. But $\left(\phi^{\prime}, f\right)=\left(\phi^{\prime}, T_{s} f\right)$ by (c) of Definition 3 , hence $\left(\phi^{\prime}, f\right)=\left(\phi^{\prime}, \sum a_{i} T_{s_{i}} f\right)$ for any convex combination of these. Thus for any $\alpha \in R^{+},\left(\phi^{\prime}, f\right)$ $=\left(\phi^{\prime}, T_{\alpha} f\right)$, and $\left(\phi^{\prime}, f\right)=\lim _{\alpha}\left(\phi^{\prime}, T_{\alpha} f\right)=\left(\phi^{\prime}, \lim _{\alpha} T_{\alpha} f\right)=\left(\phi^{\prime}, m(f) f_{1}\right)$ $=m(f)$ by $(\mathrm{b})$ of Definition 3 .

\section{BIBLIOGRAPHY}

1. S. Banach, Théorie des opérations linéaires, Warsaw, 1932.

2. W. F. Eberlein, Abstract ergodic theorems and weakly almost periodic functions, Trans. Amer. Math. Soc. vol. 67 (1949) pp. 217-240.

3. M. Jerison, $A$ property of extreme points of compact convex sets, Proc. Amer. Math. Soc. vol. 5 (1954) pp. 782-783.

4. J. L. Kelley, General topology, New York. 1955.

5. G. G. Lorentz, A contribution to the theory of divergent sequences, Acta Math. vol. 80 (1948) pp. 167-190.

UNIVERSITY OF MichigAN,

UNIVERSITY OF ROCHESTER AND

YALE UNIVERSITY 